INLO-PUB-5/93

\title{
Large momentum expansion of two-loop self-energy diagrams with arbitrary masses
}

\author{
A. I. Davydychev*(a) , V. A. Smirnov*(b) and J. B. Tausk ${ }^{\dagger}(a)$ \\ (a) Instituut-Lorentz, University of Leiden, \\ P.O.B. 9506, 2300 RA Leiden, The Netherlands \\ (b) Institut für Theoretische Physik, Universität Göttingen \\ Bunsenstrasse 9, D-3400 Göttingen, Germany
}

February 1993

\begin{abstract}
For two-loop two-point diagrams with arbitrary masses, an algorithm to derive the asymptotic expansion at large external momentum squared is constructed. By using a general theorem on asymptotic expansions of Feynman diagrams, the coefficients of the expansion are calculated analytically. For some two-loop diagrams occurring in the Standard Model, comparison with results of numerical integration shows that our expansion works well in the region above the highest physical threshold.
\end{abstract}

*Permanent address: Nuclear Physics Institute, Moscow State Univ., 119899, Moscow, Russia. E-mail addresses: davyd@compnet.msu.su and smirnov@compnet.msu.su

${ }^{\dagger}$ Research supported by the Stichting FOM. E-mail address: tausk@rulgm0.leidenuniv.nl 


\section{Introduction}

The high precision that has been achieved in high energy $e^{+} e^{-}$experiments, especially at LEP, makes them sensitive to radiative corrections in the Standard Model at the one and two loop level. However, some complicated theoretical problems are connected with the evaluation of two-loop corrections involving massive internal particles (heavy quarks, $W$ and $Z$ bosons, Higgs particles) whose masses cannot be neglected in the region of interest. In particular, much attention has been paid to the evaluation of massive two-loop self-energy diagrams. In some well known special cases, such as the QED correction to the photon self-energy, they can be evaluated exactly [1, 2], and the result can be expressed in terms of trilogarithms. The problem becomes essentially more difficult when all the internal particles of the diagram are massive. Exact results for the corresponding Feynman integrals are not known, and we need to look for other approaches to calculate the contributions of such diagrams.

One of the possible ways is by numerical integration. For this purpose, it is convenient to use a two-fold integral representation [3] obtained for the so-called "master" two-loop two-point integral. Another way is by constructing asymptotic expansions of such diagrams in different regions, the coefficients of the expansions being calculated analytically. For example, in ref. 페 we constructed an expansion for the general case of the two-loop self-energy diagram that works when the external momentum squared $\left(k^{2}\right)$ is below the first physical threshold. We showed that, unless we are very close to the threshold, only a few terms of the expansion are needed to obtain highly accurate results.

In the present paper we shall consider another situation, when $k^{2}$ is larger than the highest threshold of the diagram. This case is more complicated, because the corresponding expansion is not a usual Taylor expansion, but also contains logarithms and squared logarithms of $\left(-k^{2}\right)$ (in four dimensions) yielding an imaginary part when the momentum is time-like. The procedure of calculating this diagram in the form of a series in inverse powers of the external momentum (plus logarithms) resembles the standard procedure of analytic continuation of the hypergeometric function (some explicit examples of such a procedure connected with Feynman integrals were presented e.g. in ref. [5]). To obtain this expansion, we shall apply a general mathematical theorem on asymptotic expansions of Feynman integrals in the limit of large momenta. This theorem holds at least in the case when the external momenta are not restricted to a mass shell. However, in our case of a two-point massive diagram, it is valid for any values of the external momentum. All the coefficients of the expansion have a natural interpretation in terms of Feynman integrals and are analytically calculable

in the case we consider. Expansions of this kind were presented in refs. [6, 7, 8, 9] and rigorously proved in ref. [10 (see also ref. [11] for a review).

The remainder of this paper is organized as follows. In section 2 we explain how the general theorem can be applied to construct the large momentum expansion for the general mass case of two-loop self-energies. We present an algorithm by which any term of this expansion can be calculated analytically. In section 3 we discuss the analytical results obtained for the coefficients. Section 4 is devoted to a comparison of the asymptotic expansion with the results of a numerical integration using the method 
of ref. [3]. Section 5 contains our conclusions. Finally, a number of formulae we need for massless two-point integrals and for massive vacuum integrals are collected in appendices $\mathrm{A}$ and $\mathrm{B}$, respectively.

\section{Constructing the asymptotic expansion}

We shall consider the "master" two-loop self-energy diagram presented in Fig.1a. The corresponding Feynman integral can be defined as

$$
J\left(\left\{\nu_{i}\right\} ;\left\{m_{i}\right\} ; k\right)=\iint \frac{\mathrm{d}^{n} p \mathrm{~d}^{n} q}{D_{1}^{\nu_{1}} D_{2}^{\nu_{2}} D_{3}^{\nu_{3}} D_{4}^{\nu_{4}} D_{5}^{\nu_{5}}},
$$

where $D_{i}=\left(p_{i}^{2}-m_{i}^{2}+i 0\right)$ are massive denominators (for brevity, we shall omit the "causal" $i 0$ 's below), $\nu_{i}$ are the powers of these denominators, $n=4-2 \varepsilon$ is the space-time dimension (in the framework of dimensional regularization [12, 13]), and the momenta of the lines $p_{i}$ are constructed from the external momentum $k$ and the loop integration momenta $p$ and $q$ (with due account of the momentum conservation in all vertices). Two-loop diagrams with three or four internal lines correspond to the cases when some of the $\nu$ 's in eq. (11) are equal to zero. Moreover, in the case of integer $\nu$ 's the two-loop diagram with another topology (see Fig.1b) can also be reduced to integrals (1), if we use the decomposition of first and fourth denominators (see e.g. in 14 ). So, if we deal with integer powers of denominators, it is in general sufficient to consider the integrals (1) with different powers $\nu_{i}$.

Now let us introduce the result that the asymptotic expansion theorem gives for our case (the general case of this theorem can be found in ref. [11]):

$$
J_{\Gamma} \rightleftharpoons \sum_{\gamma} J_{\Gamma / \gamma} \circ \mathcal{T}_{\left\{m_{i}\right\} ;\left\{q_{i}\right\}} J_{\gamma}
$$

where $\Gamma$ is the main graph (see Fig.1a), $\gamma$ are subgraphs involved in the asymptotic expansion (see below), $\Gamma / \gamma$ is the reduced graph obtained from $\Gamma$ by shrinking the subgraph $\gamma$ to a single point, $J_{\gamma}$ denotes the dimensionally-regularized Feynman integral corresponding to a graph $\gamma$ (for example, $J_{\Gamma}$ is given by (11) $), \mathcal{T}_{\left\{m_{i}\right\} ;\left\{q_{i}\right\}}$ is the operator of Taylor expansion of the integrand in masses and "small" momenta $q_{i}$

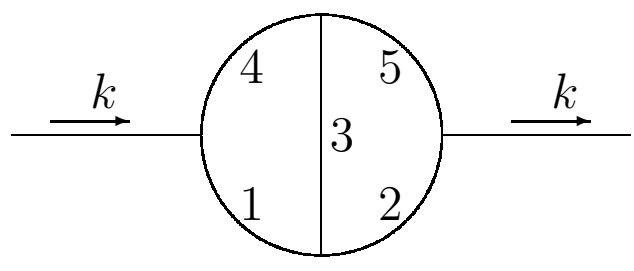

(a)

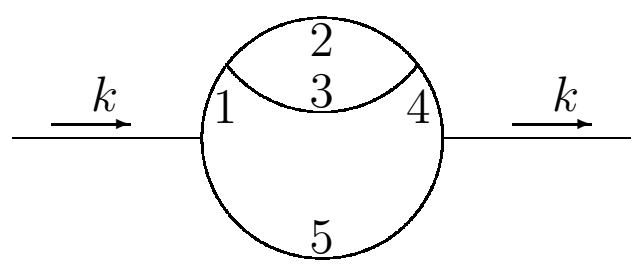

(b)

Figure 1: Two-loop self-energy diagrams 
(that are "external" for the given subgraph $\gamma$, but do not contain the "large" external momentum $k$ ), and the symbol "०" means that the resulting polynomial in these momenta should be inserted into the numerator of the integrand of $J_{\Gamma / \gamma}$. It is implied that the operator $\mathcal{T}$ acts on the integrands before the loop integrations are performed.

In our case, the sum (2) goes over all subgraphs $\gamma$ that become one-particle irreducible when we connect the two vertices with external momentum $k$ by a line. In other words, we should consider all possibilities to "distribute" the external momentum $k$ among $p_{i}$, and for each arrangement the subgraph $\gamma$ will coincide with the subset of lines involving $k$, while lines without $k$ should be removed. For our graph $\Gamma$ (see Fig.1a), all possible subgraphs $\gamma$ (there are five different types of them) are presented in Fig.2 (dotted lines correspond to the lines that do not belong to $\gamma)$

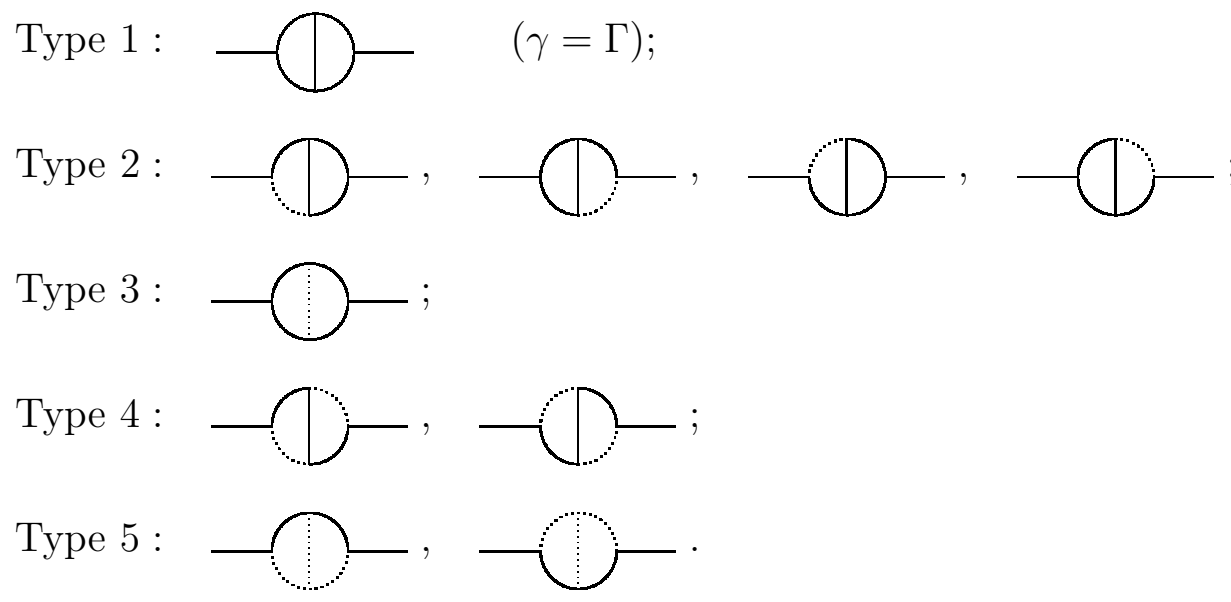

Figure 2: The subgraphs $\gamma$ contributing to the large $k^{2}$ expansion

The reduced graphs $\Gamma / \gamma$ correspond to the dotted lines and can be obtained by shrinking all solid lines to a point. In such a way, we obtain that for the second and third type (see Fig.2) $J_{\Gamma / \gamma}$ corresponds to a massive tadpole, for the fourth type we obtain a product of two massive tadpoles, while for the fifth type we get a two-loop massive vacuum integral (with three internal lines).

The Taylor expansion operator $\mathcal{T}$ expands the denominators of the integrand in the following way:

$$
\begin{gathered}
\mathcal{T}_{m} \frac{1}{\left[p^{2}-m^{2}\right]^{\nu}}=\frac{1}{\left[p^{2}\right]^{\nu}} \sum_{j=0}^{\infty} \frac{(\nu)_{j}}{j !}\left(\frac{m^{2}}{p^{2}}\right)^{j} \\
\mathcal{T}_{m ; q} \frac{1}{\left[(k-q)^{2}-m^{2}\right]^{\nu}}=\frac{1}{\left[k^{2}\right]^{\nu}} \sum_{j=0}^{\infty} \frac{(\nu)_{j}}{j !}\left(\frac{2(k q)-q^{2}+m^{2}}{k^{2}}\right)^{j},
\end{gathered}
$$

where

$$
(\nu)_{j} \equiv \frac{\Gamma(\nu+j)}{\Gamma(\nu)}
$$


is the Pochhammer symbol. In fact, on the r.h.s. of (团) it is understood that the real expansion goes over the total power of $m$ and $q$ (so, for example, the terms $q^{2}, m^{2}$ and $(k q)^{2} / k^{2}$ should be considered together). The reason is that when we evaluate the massive vacuum integrals $J_{\Gamma / \gamma}$ with these momenta $q$ in the numerator, the powers of $q$ will be transformed into powers of the masses. If we have several denominators to expand, then we should also collect all terms with the same total power of masses and "small" momenta $q_{i}$.

Now we are able to consider what integrals correspond to different terms of the asymptotic expansion (see Fig.2).

1. In this case, $\gamma=\Gamma$. All denominators of (11) should be expanded in masses,

$$
\mathcal{T}_{\left\{m_{i}\right\}} J\left(\left\{\nu_{i}\right\} ;\left\{m_{i}\right\} ; k\right)=\sum_{j_{1}, \ldots, j_{5}=0}^{\infty} \frac{\left(\nu_{1}\right)_{j_{1}} \ldots\left(\nu_{5}\right)_{j_{5}}}{j_{1} ! \ldots j_{5} !}\left(m_{1}\right)^{j_{1}} \ldots\left(m_{5}\right)^{j_{5}} J\left(\left\{\nu_{i}+j_{i}\right\} ;\{0\} ; k\right) .
$$

Note that if we consider the case $\nu_{1}=\ldots=\nu_{5}=1$, the first term of the expansion (6) (with $j_{1}=\ldots=j_{5}=0$ ) gives the well-known result: $-6 \zeta(3) \pi^{4} / k^{2}$. Two-loop massless integrals with higher integer powers of denominators occurring on the r.h.s. of (6) can be evaluated by use of the integration-by-parts technique [14] (see Appendix A).

2. Let us consider only the first contribution of the second type (see Fig.2), when $\gamma$ is obtained from $\Gamma$ by removing line 1 . Then we get

$$
\int \frac{\mathrm{d}^{n} p}{\left[p^{2}-m_{1}^{2}\right]^{\nu_{1}}} \mathcal{T}_{m_{2}, \ldots, m_{5} ; p} \int \frac{\mathrm{d}^{n} q}{\left[q^{2}-m_{2}^{2}\right]^{\nu_{2}}\left[(p-q)^{2}-m_{3}^{2}\right]^{\nu_{3}}\left[(k-p)^{2}-m_{4}^{2}\right]^{\nu_{4}}\left[(k-q)^{2}-m_{5}^{2}\right]^{\nu_{5}}} .
$$

After expanding the integrand of the $q$-integral in masses and $p$, we obtain products of massless one-loop integrals (see Appendix A) and massive tadpoles with numerators that can be calculated by use of eq. (34) (see Appendix B). Other contributions of the second type can be evaluated in the same way.

3. In the case when the central line is removed from $\gamma$, we obtain:

$$
\begin{aligned}
& \int \frac{\mathrm{d}^{n} p}{\left[p^{2}-m_{3}^{2}\right]^{\nu_{3}}} \\
& \quad \times \mathcal{T}_{m_{1}, m_{2}, m_{4}, m_{5} ; p} \int \frac{\mathrm{d}^{n} q}{\left[(k+p-q)^{2}-m_{1}^{2}\right]^{\nu_{1}}\left[(k-q)^{2}-m_{2}^{2}\right]^{\nu_{2}}\left[(p-q)^{2}-m_{4}^{2}\right]^{\nu_{4}}\left[q^{2}-m_{5}^{2}\right]^{\nu_{5}}} .
\end{aligned}
$$

After expansion, we obtain integrals of the same type as in the previous case.

4. There are no loop integrations in the subgraph $\gamma$, and we get for the first contribution of the fourth type:

$$
\begin{aligned}
\iint \frac{\mathrm{d}^{n} p}{\left[p^{2}-m_{1}^{2}\right]^{\nu_{1}}} \frac{\mathrm{d}^{n} q}{\left[q^{2}-m_{5}^{2}\right]^{\nu_{5}}} \\
\quad \times \mathcal{T}_{m_{2}, m_{3}, m_{4} ; p, q}\left(\frac{1}{\left[(k-q)^{2}-m_{2}^{2}\right]^{\nu_{2}}\left[(k-p-q)^{2}-m_{3}^{2}\right]^{\nu_{3}}\left[(k-p)^{2}-m_{4}^{2}\right]^{\nu_{4}}}\right) .
\end{aligned}
$$

As a result, we obtain products of two one-loop tadpoles with numerators (also for the second contribution), which can be evaluated by use of eq. (35) (see Appendix B). 
5. In this case we obtain a non-trivial two-loop vacuum integral. For example, the first contribution of the fifth type gives:

$$
\begin{aligned}
\iint \frac{\mathrm{d}^{n} p}{\mathrm{~d}^{n} q} & \\
& \left.\times \mathrm{p}^{2}-m_{1}^{2}\right]^{\nu_{1}}\left[q^{2}-m_{2}^{2}\right]^{\nu_{2}}\left[(p-q)^{2}-m_{3}^{2}\right]^{\nu_{3}} ;, q \\
&
\end{aligned}
$$

Expanding the denominators, we obtain two-loop vacuum integrals with numerators, that can be evaluated by use of eq. (36) presented in Appendix B (the same for the second contribution of the fifth type). Note, that here we obtain the same two-loop vacuum integrals as those involved in the small- $k^{2}$ expansion (see [4). In particular, for unit powers of denominators the dependence on masses can be expressed in terms of dilogarithms (see (29)-(33)).

So, the total asymptotic expansion is the sum of all terms presented, and we see that all integrals corresponding to the coefficients of the expansion can be evaluated.

\section{Analytical results}

In principle, eqs. (6)-(10) presented in the previous section (together with the formulae of Appendices A and B) enable one to construct analytical expressions for the coefficients of the large- $k^{2}$ expansion. However, in the general case of unequal masses the higher-order coefficients become rather cumbersome. To calculate these coefficients, we used the REDUCE system [15]. The algorithm constructed is applicable to integrals with arbitrary values of masses, space-time dimension and (integer) powers of denominators. If we are interested in the result near $n=4$, we perform an expansion in $\varepsilon=(4-n) / 2$ to get the divergent and finite parts of the coefficients.

One of the most important examples is the "master" two-loop diagram (presented in Fig.1a) in the case $\nu_{1}=\ldots=\nu_{5}=1$. In this case, the result should be finite as $n \rightarrow 4$ (and it is a non-trivial check of the algorithm that all the divergent contributions from separate terms of (6)-(10) cancel in this sum !). A rigorous proof

of the finiteness of the expansion was given in [9] (it was based on the so-called $R^{*}$-operation [16]).

Let us define

$$
\begin{aligned}
\left.J\left(1, \ldots, 1 ; m_{1}, \ldots, m_{5} ; k\right)\right|_{n=4} & \equiv J\left(m_{1}, \ldots, m_{5} ; k\right) \\
& \equiv-\frac{\pi^{4}}{k^{2}} \mathcal{M}\left(m_{1}, \ldots, m_{5} ; k\right) \equiv-\frac{\pi^{4}}{k^{2}} \sum_{j=0}^{\infty} \frac{\mathcal{M}_{j}}{\left(k^{2}\right)^{j}},
\end{aligned}
$$

where the coefficient functions $\mathcal{M}_{j}$ include the powers of masses and the logarithms of masses and momentum squared. It is easy to see that the only integral contributing to $\mathcal{M}_{0}$ is $J^{(0)}(1,1,1,1,1)$ in (6) (see eq. (26) in Appendix A). So we get the obvious result that the expansion starts from

$$
\mathcal{M}_{0}=6 \zeta(3)
$$


The $\mathcal{M}_{1}$ term already includes contributions of all terms (6)-(10) (with the exception of (9) that begins to contribute starting from $\mathcal{M}_{2}$ ); this yields

$$
\begin{aligned}
\mathcal{M}_{1}= & \frac{m_{1}^{2}}{2}\left\{\ln ^{2}\left(-\frac{k^{2}}{m_{1}^{2}}\right)+4 \ln \left(-\frac{k^{2}}{m_{1}^{2}}\right)-\ln \frac{m_{2}^{2}}{m_{1}^{2}} \ln \frac{m_{3}^{2}}{m_{1}^{2}}+6\right\} \\
& +\left\{\text { analogous terms with } m_{2}^{2}, m_{4}^{2}, m_{5}^{2}\right\} \\
& +\frac{m_{3}^{2}}{2}\left\{2 \ln ^{2}\left(-\frac{k^{2}}{m_{3}^{2}}\right)+4 \ln \left(-\frac{k^{2}}{m_{3}^{2}}\right)-\ln \frac{m_{1}^{2}}{m_{3}^{2}} \ln \frac{m_{2}^{2}}{m_{3}^{2}}-\ln \frac{m_{4}^{2}}{m_{3}^{2}} \ln \frac{m_{5}^{2}}{m_{3}^{2}}\right\} \\
& +\frac{1}{2}\left\{F\left(m_{1}^{2}, m_{2}^{2}, m_{3}^{2}\right)+F\left(m_{4}^{2}, m_{5}^{2}, m_{3}^{2}\right)\right\}
\end{aligned}
$$

where the symmetric function $F$ is defined by (30)-(33) (note that $F$ has the dimension of mass squared). We see that $\mathcal{M}_{1}$ contains also $\ln \left(-k^{2}\right)$ and $\ln ^{2}\left(-k^{2}\right)$ terms, which means that our expansion (at $n=4$ ) is not a usual Taylor expansion. In fact, the highest power of $\ln \left(-k^{2}\right)$ is connected with the highest order of pole in $\varepsilon$ (double pole) that can occur in the two-loop integrals involved. Note that for positive $k^{2}$ the sign of the imaginary part produced by these logarithms is defined by the "causal" $i 0$-prescription,

$$
\ln \left(-k^{2}-i 0\right)=\ln \left(k^{2}\right)-i \pi \quad\left(k^{2}>0\right)
$$

Let us also present the result for the next term of the expansion,

$$
\begin{aligned}
\mathcal{M}_{2}= & \frac{m_{1}^{4}}{8}\left\{2 \ln ^{2}\left(-\frac{k^{2}}{m_{1}^{2}}\right)+4 \ln \left(-\frac{k^{2}}{m_{1}^{2}}\right)-2 \ln \frac{m_{2}^{2}}{m_{1}^{2}} \ln \frac{m_{3}^{2}}{m_{1}^{2}}+7\right\} \\
& +\left\{\text { analogous terms with } m_{2}^{4}, m_{4}^{4}, m_{5}^{4}\right\} \\
+ & \frac{m_{3}^{4}}{4}\left\{-2 \ln ^{2}\left(-\frac{k^{2}}{m_{3}^{2}}\right)-2 \ln \left(-\frac{k^{2}}{m_{3}^{2}}\right)+\ln \frac{m_{1}^{2}}{m_{3}^{2}} \ln \frac{m_{2}^{2}}{m_{3}^{2}}+\ln \frac{m_{4}^{2}}{m_{3}^{2}} \ln \frac{m_{5}^{2}}{m_{3}^{2}}+6\right\} \\
- & \frac{1}{2}\left(m_{1}^{2} m_{2}^{2}+m_{4}^{2} m_{5}^{2}\right) \\
+ & \frac{m_{1}^{2} m_{4}^{2}}{2}\left\{\ln ^{2}\left(-\frac{k^{2}}{m_{1}^{2}}\right)+\ln \left(-\frac{k^{2}}{m_{4}^{2}}\right)+4 \ln \left(-\frac{k^{2}}{m_{1}^{2}}\right)+4 \ln \left(-\frac{k^{2}}{m_{4}^{2}}\right)\right. \\
& \left.-\quad \ln \frac{m_{2}^{2}}{m_{1}^{2}} \ln \frac{m_{3}^{2}}{m_{1}^{2}}-\ln \frac{m_{3}^{2}}{m_{4}^{2}} \ln \frac{m_{5}^{2}}{m_{4}^{2}}+8\right\} \\
+ & \frac{m_{1}^{2} m_{5}^{2}}{2}\left\{2 \ln \ln ^{2}\left(-\frac{k^{2}}{m_{1}^{2}}\right)+2 \ln \ln ^{2}\left(-\frac{k^{2}}{m_{5}^{2}}\right)+2 \ln \left(-\frac{k^{2}}{m_{1}^{2}}\right)+2 \ln \left(-\frac{k^{2}}{m_{5}^{2}}\right)\right. \\
& \left.-\ln \frac{m_{2}^{2}}{m_{1}^{2}} \ln \frac{m_{3}^{2}}{m_{1}^{2}}-\ln \frac{m_{3}^{2}}{m_{5}^{2}} \ln \frac{m_{4}^{2}}{m_{5}^{2}}-\ln \frac{m_{1}^{2}}{m_{5}^{2}}+2\right\}
\end{aligned}
$$




$$
\begin{gathered}
+\left\{\text { analogous term with } m_{2}^{2} m_{4}^{2}\right\} \\
+\frac{m_{1}^{2} m_{3}^{2}}{2}\left\{2 \ln ^{2}\left(-\frac{k^{2}}{m_{3}^{2}}\right)-2 \ln \left(-\frac{k^{2}}{m_{1}^{2}}\right)-\ln \frac{m_{1}^{2}}{m_{3}^{2}} \ln \frac{m_{2}^{2}}{m_{3}^{2}}-\ln \frac{m_{4}^{2}}{m_{3}^{2}} \ln \frac{m_{5}^{2}}{m_{3}^{2}}-8\right\} \\
+\left\{\text { analogous terms with } m_{2}^{2} m_{3}^{2}, m_{4}^{2} m_{3}^{2}, m_{5}^{2} m_{3}^{2}\right\} \\
+\frac{1}{4}\left\{\left(m_{1}^{2}+m_{2}^{2}-m_{3}^{2}+2 m_{4}^{2}+2 m_{5}^{2}\right) F\left(m_{1}^{2}, m_{2}^{2}, m_{3}^{2}\right)\right. \\
\left.+\left(2 m_{1}^{2}+2 m_{2}^{2}-m_{3}^{2}+m_{4}^{2}+m_{5}^{2}\right) F\left(m_{4}^{2}, m_{5}^{2}, m_{3}^{2}\right)\right\} .
\end{gathered}
$$

Higher contributions are more cumbersome, and we do not present them here (but we are going to use them below, when comparing our expansion with the results of numerical integration). By use of the REDUCE system [15], for the general massive case of the integral (11) (when all five masses are arbitrary) we obtained analytical results for the coefficient functions up to $\mathcal{M}_{6}$.

There are also some other possibilities to check the correctness of our results (in addition to cancellation of $1 / \varepsilon$ poles). For example, in ref. [2] analogous results were presented for some special cases when some of the masses are zero while others are equal (see also ref. [17] where these results were generalised to the case of arbitrary space-time dimension $n$ ). In these cases, we compared our results for the coefficients (at $n=4)$ and found complete agreement.

\section{Numerical results}

In this section, we will continue to focus on the "master" two-loop integral corresponding to Fig. 1a. In general, it has two two-particle thresholds, at $k^{2}=\left(m_{1}+m_{4}\right)^{2}$ and $k^{2}=\left(m_{2}+m_{5}\right)^{2}$, and two three-particle thresholds at $k^{2}=\left(m_{1}+m_{3}+m_{5}\right)^{2}$ and $k^{2}=\left(m_{2}+m_{3}+m_{4}\right)^{2}$. The asymptotic expansion theorem quoted in (2) provides a series of approximations to this integral of the form (see eq. (11)):

$$
\mathcal{M}^{(N)} \equiv \sum_{j=0}^{N} \frac{\mathcal{M}_{j}}{\left(k^{2}\right)^{j}},
$$

such that the remainder behaves like

$$
\mathcal{M}-\mathcal{M}^{(N)}=\mathcal{O}\left(\left(k^{2}\right)^{-N-1} \ln ^{2}\left(-k^{2}\right)\right)
$$

as $k^{2} \rightarrow \infty$

Strictly speaking, this does not imply convergence of the series for any fixed value of $k^{2}$, but from experience with special cases where exact analytical results are known, one would expect the series to converge when $\left|k^{2}\right|$ is larger than the highest threshold. In order to see whether this is true in the general case, and whether the asymptotic expansion can be used as a practical means of calculating these integrals, we made some numerical comparisons for two physical examples. 
The first example is:

$$
J\left(M_{W}, M_{W}, M_{Z}, m_{b}, m_{b} ; k\right),
$$

where the $M_{W}, M_{Z}$ and $m_{b}$ denote the masses of the $W$-boson, the $Z$-boson and the bottom quark. This integral is a contribution to the top quark self-energy. In this case, both the two-particle thresholds coincide. The three-particle thresholds also coincide with each other.

The other example occurs in the photon, the Higgs and the Z-boson self-energies:

$$
J\left(m_{t}, m_{t}, M_{Z}, m_{t}, m_{t} ; k\right) .
$$

Here $m_{t}$ is the top quark mass. As in the first example, there are only two distinct thresholds. We calculated (18) and (19) numerically using the method of ref. [3]. The values we took for the masses were:

$$
M_{Z}=91 \mathrm{GeV}, \quad M_{W}=80 \mathrm{GeV}, \quad m_{t}=140 \mathrm{GeV}, \quad m_{b}=5 \mathrm{GeV} .
$$

The results are displayed in Figs. 3 and 4 . In each plot, the first threshold is exactly on the left edge and the position of the highest threshold is indicated by a dashed vertical line. The dotted horizontal line shows the lowest order asymptotic approximation $\mathcal{M}^{(0)} \equiv \mathcal{M}_{0}=6 \zeta(3)=7.21234 \ldots$ (the imaginary part is zero to this order). The curves labeled $1,2, \ldots$ show the approximations $\mathcal{M}^{(1)}, \mathcal{M}^{(2)}, \ldots$ defined by eq. (16). The results of the numerical integration program are shown as crosses.

At large values of $k^{2}$, say ten times the highest threshold or higher, $\mathcal{M}^{(3)}$ already agrees with the numerical results to within $0.01 \%$, which is the order of magnitude of the error in the numerical results.

The most interesting region is immediately above the highest threshold, where we would still expect the expansion to converge, but more slowly than at large $k^{2}$. In fact, in our first example $(18), \mathcal{M}^{(4)}$ is still accurate to within $1 \%$ on the threshold itself. In the second example (19), the convergence near the threshold is less rapid. This region is shown in more detail in Fig. 5, where the left edge of the plots corresponds to the highest threshold. On the threshold itself, the error in $\mathcal{M}^{(6)}$ is about $6 \%$ (in the real part), but it drops down to less than $1 \%$ by the time $k^{2}$ is a factor of 1.2 above the threshold.

Note that in [18] radiative corrections to the on-shell $H \rightarrow b \bar{b}$ squared amplitude (represented by a diagram like Fig.1a) were examined, and it was pointed out that the mass correction $\mathcal{M}_{1}$ essentially improved the description above the last threshold.

Finally, when we go below the highest threshold, we see large deviations between the asymptotic approximations and the numerical values, and the series ceases to converge. From this we see that the asymptotic expansion can only be applied to the region above the highest threshold, as expected.

\section{Conclusions}

Thus, in the present paper we considered an algorithm to construct an asymptotic expansion of two-loop self-energy diagrams (see Fig.1) for large values of $k^{2}$. This 
algorithm can be applied to the general case of different masses, and integer powers of denominators. We used the asymptotic expansion theorem (2) to derive the different contributions to the expansion (see (6) $-(10)$ ). In any order, the coefficients of the expansion can be expressed in terms of known one- and two-loop propagator-type massless integrals, and one- and two-loop massive vacuum integrals.

By use of the REDUCE system, we wrote a program that automatically generates analytical expressions for the coefficients of the asymptotic expansion. Then we considered the "master" two-loop diagram with different masses (Fig.1a), and we obtained expressions for the terms of the asymptotic expansion of the integral (11) up to $1 /\left(k^{2}\right)^{7}$ terms (with logarithms). The first three coefficient functions $\mathcal{M}_{0,1,2}$ are given by equations (12), (13), (15). For some concrete diagrams occurring in the Standard Model, we made a numerical comparison with the results of a two-fold numerical integration based on the algorithm of [3]. We found that in the region above the highest physical threshold of the diagram, our expansion converges well, and in the region not very close to the threshold it is sufficient to take only a few terms of the expansion to provide a good accuracy.

Thus, the present paper (together with [4]) solves the problem of constructing asymptotic expansions of massive two-loop self-energy diagrams in the limits of small and large $k^{2}$ values (when we are either below the lowest physical threshold or above the highest one). The analytical description of the behaviour between the thresholds still remains a problem. Note, however, that the general asymptotic expansion theorem can also be used for other limits in order to calculate the considered diagram in situations when some of the masses are large and the others are small.

A procedure analogous to the one considered here can be also applied to threepoint two-loop vertex diagrams, when all external lines are above the corresponding thresholds (and also in some other cases). Note that some two-loop massless integrals with off-shell external momenta (needed for such an expansion) were calculated in ref. [19. For numerical comparison, a parametric integral representation obtained in ref. 20] can be used.

\section{Acknowledgements}

We are grateful to F.A. Berends and W.L. van Neerven for their assistance and helpful discussions. We are also grateful to D.J. Broadhurst, R. Buchert, A.L. Kataev and D. Kreimer for useful discussions, and to H. Kuijf and S.C. van der Marck for writing the program that calculates the integral (11) numerically. One of the authors (A.D.) is grateful to Instituut-Lorentz for their hospitality and to the Nederlandse organisatie voor Wetenschappelijk Onderzoek (NWO) for financial support. Another author (V.S.) is thankful to the Institute for Theoretical Physics of Göttingen University for kind hospitality and to the Heraeus Foundation for support. 


\section{Appendix A: Massless integrals}

Here we present some formulae needed for evaluation of one- and two-loop massless integrals occurring in the paper. These results are well known, and we write them only for completeness.

The massless one-loop integral is

$$
\begin{aligned}
I^{(0)}\left(\nu_{1}, \nu_{2}\right) & \equiv \int \frac{\mathrm{d}^{n} p}{\left[p^{2}\right]^{\nu_{1}}\left[(k-p)^{2}\right]^{\nu_{2}}} \\
& =i^{1-n} \pi^{n / 2}\left(k^{2}\right)^{n / 2-\nu_{1}-\nu_{2}} \frac{\Gamma\left(n / 2-\nu_{1}\right) \Gamma\left(n / 2-\nu_{2}\right) \Gamma\left(\nu_{1}+\nu_{2}-n / 2\right)}{\Gamma\left(\nu_{1}\right) \Gamma\left(\nu_{2}\right) \Gamma\left(n-\nu_{1}-\nu_{2}\right)},
\end{aligned}
$$

where $n=4-2 \varepsilon$ is the space-time dimension.

Massless two-loop integrals are defined by (see (11))

$$
J^{(0)}\left(\nu_{1}, \nu_{2}, \nu_{3}, \nu_{4}, \nu_{5}\right) \equiv J\left(\left\{\nu_{i}\right\} ;\{0\} ; k\right)
$$

By use of symmetry properties of the diagram in Fig.1a, and the integration by parts formula [14],

$$
\begin{aligned}
J^{(0)} & \left(\nu_{1}, \nu_{2}, \nu_{3}, \nu_{4}, \nu_{5}\right)=\left(\nu_{1}+2 \nu_{3}+\nu_{4}-n\right)^{-1} \\
& \times\left\{\nu_{1}\left[J^{(0)}\left(\nu_{1}+1, \nu_{2}-1, \nu_{3}, \nu_{4}, \nu_{5}\right)-J^{(0)}\left(\nu_{1}+1, \nu_{2}, \nu_{3}-1, \nu_{4}, \nu_{5}\right)\right]\right. \\
& \left.+\nu_{4}\left[J^{(0)}\left(\nu_{1}, \nu_{2}, \nu_{3}, \nu_{4}+1, \nu_{5}-1\right)-J^{(0)}\left(\nu_{1}, \nu_{2}, \nu_{3}-1, \nu_{4}+1, \nu_{5}\right)\right]\right\},
\end{aligned}
$$

these integrals (with positive integer $\nu$ 's) can be reduced to the following "boundary" integrals:

$$
\begin{gathered}
J^{(0)}\left(\nu_{1}, \nu_{2}, 0, \nu_{4}, \nu_{5}\right)=I^{(0)}\left(\nu_{1}, \nu_{4}\right) I^{(0)}\left(\nu_{2}, \nu_{5}\right) \\
J^{(0)}\left(\nu_{1}, \nu_{2}, \nu_{3}, \nu_{4}, 0\right)=\left(k^{2}\right)^{\nu_{2}+\nu_{3}-n / 2} I^{(0)}\left(\nu_{2}, \nu_{3}\right) I^{(0)}\left(\nu_{1}+\nu_{2}+\nu_{3}-n / 2, \nu_{4}\right) .
\end{gathered}
$$

For example,

$$
J^{(0)}(1,1,1,1,1)=-\frac{\pi^{4}}{k^{2}} 6 \zeta(3)+\mathcal{O}(\varepsilon)
$$

(note that $\zeta(3)$ does not occur in the divergent and finite parts of any other integrals $J^{(0)}$ with positive $\nu$ 's).

\section{Appendix B: Massive vacuum integrals}

The result for one-loop massive tadpole integral is well known [12] :

$$
K(\nu, m) \equiv \int \frac{\mathrm{d}^{n} p}{\left[p^{2}-m^{2}\right]^{\nu}}=i^{1-n} \pi^{n / 2}\left(-m^{2}\right)^{n / 2-\nu} \frac{\Gamma(\nu-n / 2)}{\Gamma(\nu)} .
$$

Two-loop vacuum massive integrals

$$
I\left(\nu_{1}, \nu_{2}, \nu_{3} ; m_{1}, m_{2}, m_{3}\right) \equiv \iint \frac{\mathrm{d}^{n} p \mathrm{~d}^{n} q}{\left[p^{2}-m_{1}^{2}\right]^{\nu_{1}}\left[q^{2}-m_{2}^{2}\right]^{\nu_{2}}\left[(p-q)^{2}-m_{3}^{2}\right]^{\nu_{3}}}
$$


have been studied e.g. in ref. [4] (some special cases were also considered in ref. [21]). For example, for unit powers of denominators we get

$$
\begin{aligned}
& I\left(1,1,1 ; m_{1}, m_{2}, m_{3}\right)=\pi^{4-2 \varepsilon} \Gamma^{2}(1+\varepsilon)\left(1+3 \varepsilon+7 \varepsilon^{2}\right) \\
& \times\left\{-\frac{1}{2 \varepsilon^{2}}\left(m_{1}^{2}+m_{2}^{2}+m_{3}^{2}\right)+\frac{1}{\varepsilon}\left(m_{1}^{2} \ln m_{1}^{2}+m_{2}^{2} \ln m_{2}^{2}+m_{3}^{2} \ln m_{3}^{2}\right)\right. \\
& -\frac{1}{2}\left[m_{1}^{2} \ln ^{2} m_{1}^{2}+m_{2}^{2} \ln ^{2} m_{2}^{2}+m_{3}^{2} \ln ^{2} m_{3}^{2}\right. \\
& \quad+\left(m_{1}^{2}+m_{2}^{2}-m_{3}^{2}\right) \ln m_{1}^{2} \ln m_{2}^{2}+\left(m_{1}^{2}-m_{2}^{2}+m_{3}^{2}\right) \ln m_{1}^{2} \ln m_{3}^{2} \\
& \left.\left.\quad+\left(-m_{1}^{2}+m_{2}^{2}+m_{3}^{2}\right) \ln m_{2}^{2} \ln m_{3}^{2}+F\left(m_{1}^{2}, m_{2}^{2}, m_{3}^{2}\right)\right]\right\}+\mathcal{O}(\varepsilon)
\end{aligned}
$$

where the function $F$ is symmetric with respect to $m_{1}^{2}, m_{2}^{2}, m_{3}^{2}$, and is defined by (see [四])

$$
F\left(m_{1}^{2}, m_{2}^{2}, m_{3}^{2}\right) \equiv m_{3}^{2} \lambda^{2}(x, y) \Phi(x, y)
$$

with

$$
\begin{gathered}
x \equiv \frac{m_{1}^{2}}{m_{3}^{2}} \quad, \quad y \equiv \frac{m_{2}^{2}}{m_{3}^{2}} \\
\lambda^{2}(x, y)=(1-x-y)^{2}-4 x y \\
\Phi(x, y)=\frac{1}{\lambda}\left\{2 \ln \left(\frac{1+x-y-\lambda}{2}\right) \ln \left(\frac{1-x+y-\lambda}{2}\right)-\ln x \ln y\right. \\
\left.-2 \operatorname{Li}_{2}\left(\frac{1+x-y-\lambda}{2}\right)-2 \operatorname{Li}_{2}\left(\frac{1-x+y-\lambda}{2}\right)+\frac{\pi^{2}}{3}\right\} .
\end{gathered}
$$

If the largest mass is $m_{1}$ or $m_{2}$, we should choose this mass as the dimensionlessmaking parameter in (30) and (31) (instead of $m_{3}$ ). In the region where $\lambda^{2}<0$, the function (30) can be represented in terms of Clausen's functions (see [4]). To obtain results for integrals (28) with higher integer powers of denominators, a recursive procedure based on integration by parts [14] can be applied (see in [4]). It is interesting that the same function (33) also occurs when we evaluate massless triangle diagrams (see e.g. in [22]; in this case $x$ and $y$ are dimensionless ratios of external momenta squared).

We also need to evaluate massive integrals with numerators. To do this, in the one-loop case we used the following explicit formula:

$$
\begin{aligned}
& \left.\int \frac{\mathrm{d}^{n} p}{\left[p^{2}-m^{2}\right]^{\nu}}\left[2\left(k_{1} p\right)\right]^{N_{1}}\left[2\left(k_{2} p\right)\right]^{N_{2}}\right|_{N_{1}+N_{2}-\text { even }}
\end{aligned}
$$

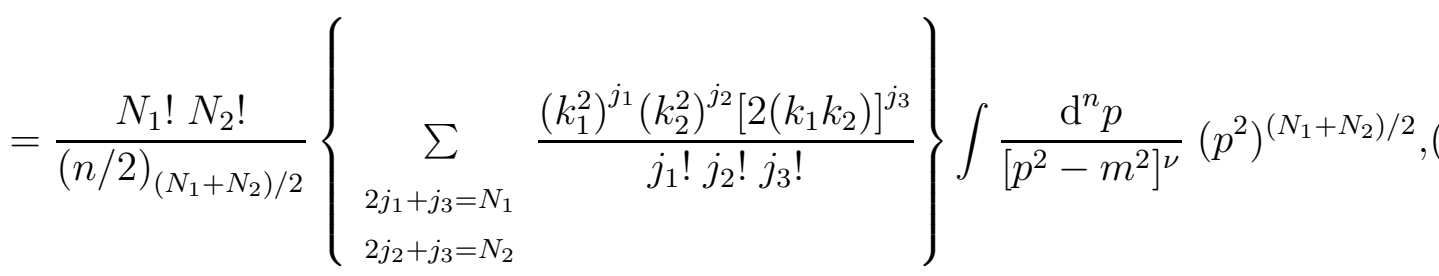


and the integral on the l.h.s. vanishes if $\left(N_{1}+N_{2}\right)$ is odd. The sum in braces goes over all non-negative integers $j_{1}, j_{2}, j_{3}$ obeying two conditions: $2 j_{1}+j_{3}=N_{1}$ and $2 j_{2}+j_{3}=N_{2}$ (so, in fact it is a one-fold finite sum). We also use a standard notation for the Pochhammer symbol (5).

When we have a two-loop integral with a numerator and two denominators (corresponding to a product of two one-loop tadpoles), the following formula can be derived:

$$
\begin{gathered}
\iint \frac{\mathrm{d}^{n} p \mathrm{~d}^{n} q}{\left[p^{2}-m_{1}^{2}\right]^{\nu_{1}}\left[q^{2}-m_{2}^{2}\right]^{\nu_{2}}}[2(k p)]^{N_{1}}[2(k q)]^{N_{2}}[2(p q)]^{N_{3}} \mid \begin{array}{c}
N_{1}+N_{3} \text { - even } \\
N_{2}+N_{3}-\text { even }
\end{array} \\
=\frac{N_{1} ! N_{2} !\left(k^{2}\right)^{\left(N_{1}+N_{2}\right) / 2}}{(n / 2)_{\left(N_{1}+N_{2}\right) / 2}}\left\{\begin{array}{c}
\sum_{\substack{2 j_{1}+j_{3}=N_{1} \\
2 j_{2}+j_{3}=N_{2}}} \frac{\left(N_{3}+j_{3}\right) !}{j_{1} ! j_{2} ! j_{3} !\left(\left(N_{3}+j_{3}\right) / 2\right) !(n / 2)_{\left(N_{3}+j_{3}\right) / 2}} \\
\times \iint \frac{\mathrm{d}^{n} p \mathrm{~d}^{n} q}{\left[p^{2}-m_{1}^{2}\right]^{\nu_{1}}\left[q^{2}-m_{2}^{2}\right]^{\nu_{2}}}\left(p^{2}\right)^{\left(N_{1}+N_{3}\right) / 2}\left(q^{2}\right)^{\left(N_{2}+N_{3}\right) / 2},
\end{array}\right.
\end{gathered}
$$

and the integral on the l.h.s. of (35) is equal to zero if $\left(N_{1}+N_{3}\right)$ or $\left(N_{2}+N_{3}\right)$ is odd. The sum in braces is of the same structure as in (34).

We also need an analogous formula for the two-loop integral with three denominators:

$$
\begin{gathered}
\left.\iint \frac{\mathrm{d}^{n} p \mathrm{~d}^{n} q}{\left[p^{2}-m_{1}^{2}\right]^{\nu_{1}}\left[q^{2}-m_{2}^{2}\right]^{\nu_{2}}\left[(p-q)^{2}-m_{3}^{2}\right]^{\nu_{3}}}[2(k p)]^{N_{1}}[2(k q)]^{N_{2}}\right|_{N_{1}+N_{2}-\text { even }} \\
=\frac{N_{1} ! N_{2} !\left(k^{2}\right)^{\left(N_{1}+N_{2}\right) / 2}}{(n / 2)\left(N_{1}+N_{2}\right) / 2} \sum_{\substack{2 j_{1}+j_{3}=N_{1} \\
2 j_{2}+j_{3}=N_{2}}} \frac{1}{j_{1} ! j_{2} ! j_{3} !} \\
\times \iint \frac{\mathrm{d}^{n} p \mathrm{~d}^{n} q}{\left[p^{2}-m_{1}^{2}\right]^{\nu_{1}}\left[q^{2}-m_{2}^{2}\right]^{\nu_{2}}\left[(p-q)^{2}-m_{3}^{2}\right]^{\nu_{3}}}\left(p^{2}\right)^{j_{1}}\left(q^{2}\right)^{j_{2}}[2(p q)]^{j_{3}}
\end{gathered}
$$

and this integral is also equal to zero if $\left(N_{1}+N_{2}\right)$ is odd.

In all formulae (34), (35) and (36), the remaining momenta in the numerators on the r.h.s. can be expressed in terms of denominators, and we arrive at the result expressed in terms of integrals (27) and (28) without numerators. 


\section{References}

[1] G. Källén and A. Sabry, Dan.Mat.Fys.Medd. 29 (1955) No.17;

J. Schwinger, Particles, sources and fields, Vol.2 (Addison-Wesley, 1973);

D.J. Broadhurst, Phys.Lett. B101 (1981) 423;

T.H. Chang, K.J.F. Gaemers and W.L. van Neerven, Nucl.Phys. B202 (1982) 407;

A. Djouadi, Nuovo Cim. 100A (1988) 357;

B.A. Kniehl, Nucl.Phys. B347 (1990) 86.

[2] D.J. Broadhurst, Z.Phys. C47 (1990) 115.

[3] D. Kreimer, Phys.Lett. B273 (1991) 277.

[4] A.I. Davydychev and J.B. Tausk, Nucl. Phys. B397 (1993), 123.

[5] E.E. Boos and A.I. Davydychev, Teor.Mat.Fiz. 89 (1991) 56 (Theor.Math.Phys. 89 (1991) 1052).

[6] F.V. Tkachov, Preprint INR P-358 (Moscow, 1984);

G.B. Pivovarov and F.V. Tkachov, Preprints INR P-0370, П-459 (Moscow, 1984).

[7] K.G. Chetyrkin and V.A. Smirnov, Preprint INR G-518 (Moscow, 1987);

K.G. Chetyrkin, Teor.Mat.Fiz. 75 (1988) 26; 76 (1988) 207.

[8] S.G. Gorishny, Nucl.Phys. B319 (1989) 633.

[9] K.G. Chetyrkin, Preprint MPI-PAE/PTh 13/91 (Munich, 1991).

[10] V.A. Smirnov, Commun.Math.Phys. 134 (1990) 109.

[11] V.A. Smirnov, Renormalization and asymptotic expansions (Birkhäuser, Basel, 1991).

[12] G. 'tHooft and M. Veltman, Nucl.Phys. B44 (1972) 189.

[13] C.G. Bollini and J.J. Giambiagi, Nuovo Cim. 12B (1972) 20.

[14] F.V. Tkachov, Phys.Lett. B100 (1981) 65;

K.G. Chetyrkin and F.V. Tkachov, Nucl.Phys. B192 (1981) 159.

[15] A.C. Hearn, REDUCE user's manual, RAND publication CP78 (Santa Monica, 1987).

[16] K.G. Chetyrkin and F.V. Tkachov, Phys.Lett. B114 (1982) 340;

K.G. Chetyrkin and V.A. Smirnov, Phys.Lett. B144 (1984) 419;

Preprint NPI MSU 89-3/80 (Moscow, 1989).

[17] D.J. Broadhurst, J. Fleischer and O.V. Tarasov, Open University preprint OUT-4102-43 (Milton Keynes, 1993). 
[18] A.L. Kataev and V.T. Kim, Preprint ENSLAPP-A-407/92 (Annecy-le-Vieux, 1992).

[19] N.I. Ussyukina and A.I. Davydychev, Phys.Lett. B298 (1993) 363; Yad.Fiz. 56, No.11 (1993) 172.

[20] D. Kreimer, Phys.Lett. B292 (1992) 341.

[21] J. van der Bij and M. Veltman, Nucl.Phys. B231 (1984) 205;

F. Hoogeveen, Nucl.Phys. B259 (1985) 19;

R. Scharf, Diploma work (Würzburg, 1991) ;

C. Ford and D.R.T. Jones, Phys.Lett. B274 (1992) 409; errata: B285 (1992) 399;

C. Ford, I. Jack and D.R.T. Jones, Nucl.Phys. B387 (1992) 373.

[22] A.I. Davydychev, J.Phys. A25 (1992) 5587. 


\section{Figure captions}

Figure 3: The real and imaginary parts of $J\left(M_{W}, M_{W}, M_{Z}, m_{b}, m_{b} ; k\right)$ for $k^{2} \geq\left(M_{W}+m_{b}\right)^{2}$. The second threshold is at $\left(M_{W}+M_{Z}+m_{b}\right)^{2}$.

Figure 4: The real and imaginary parts of $J\left(m_{t}, m_{t}, M_{Z}, m_{t}, m_{t} ; k\right)$ for $k^{2} \geq 4 m_{t}^{2}$. The second threshold is at $\left(2 m_{t}+M_{Z}\right)^{2}$.

Figure 5: The real and imaginary parts of $J\left(m_{t}, m_{t}, M_{Z}, m_{t}, m_{t} ; k\right)$ for $k^{2} \geq\left(2 m_{t}+M_{Z}\right)^{2}$. 
Fig. 3a

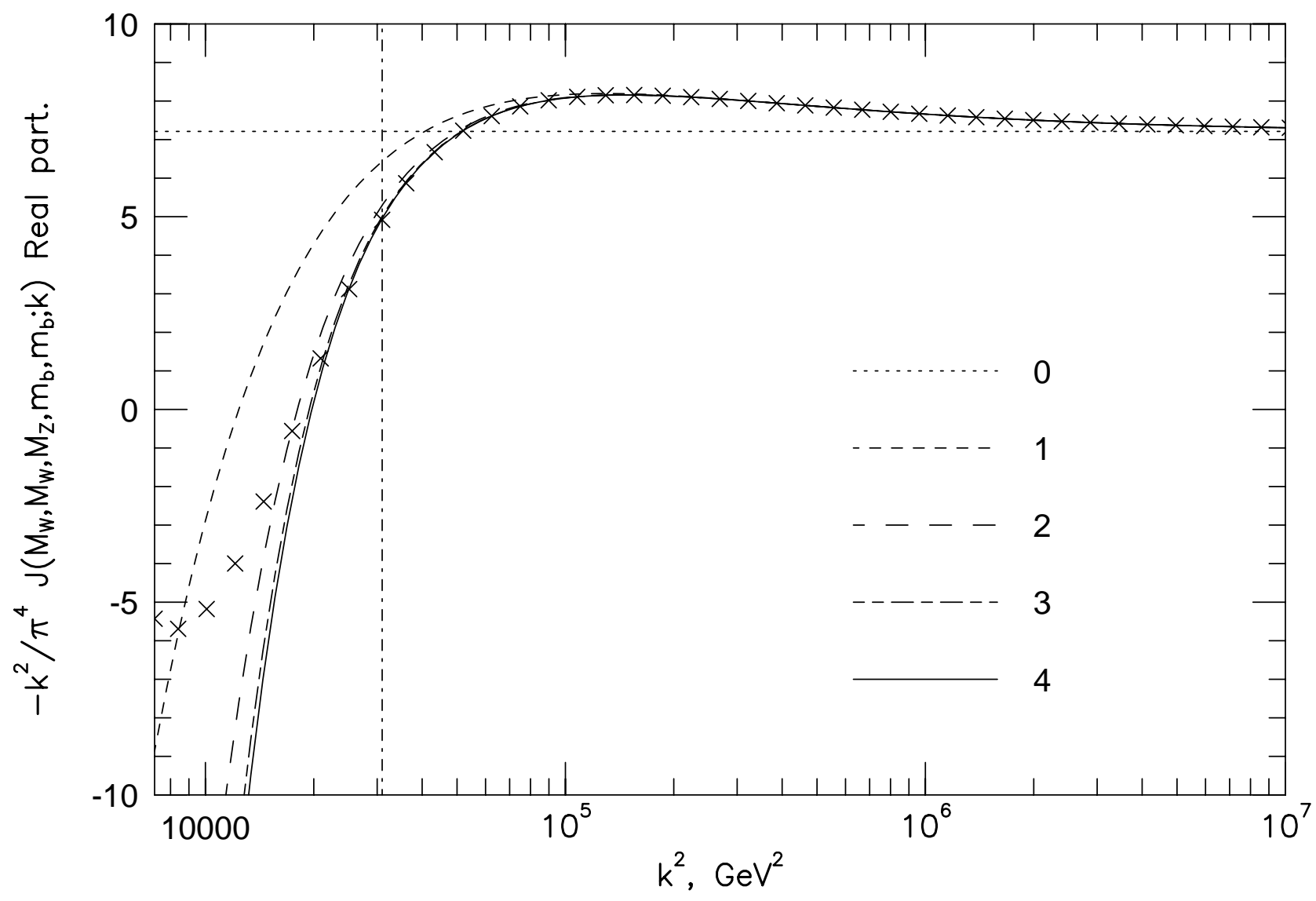

Fig. 3b

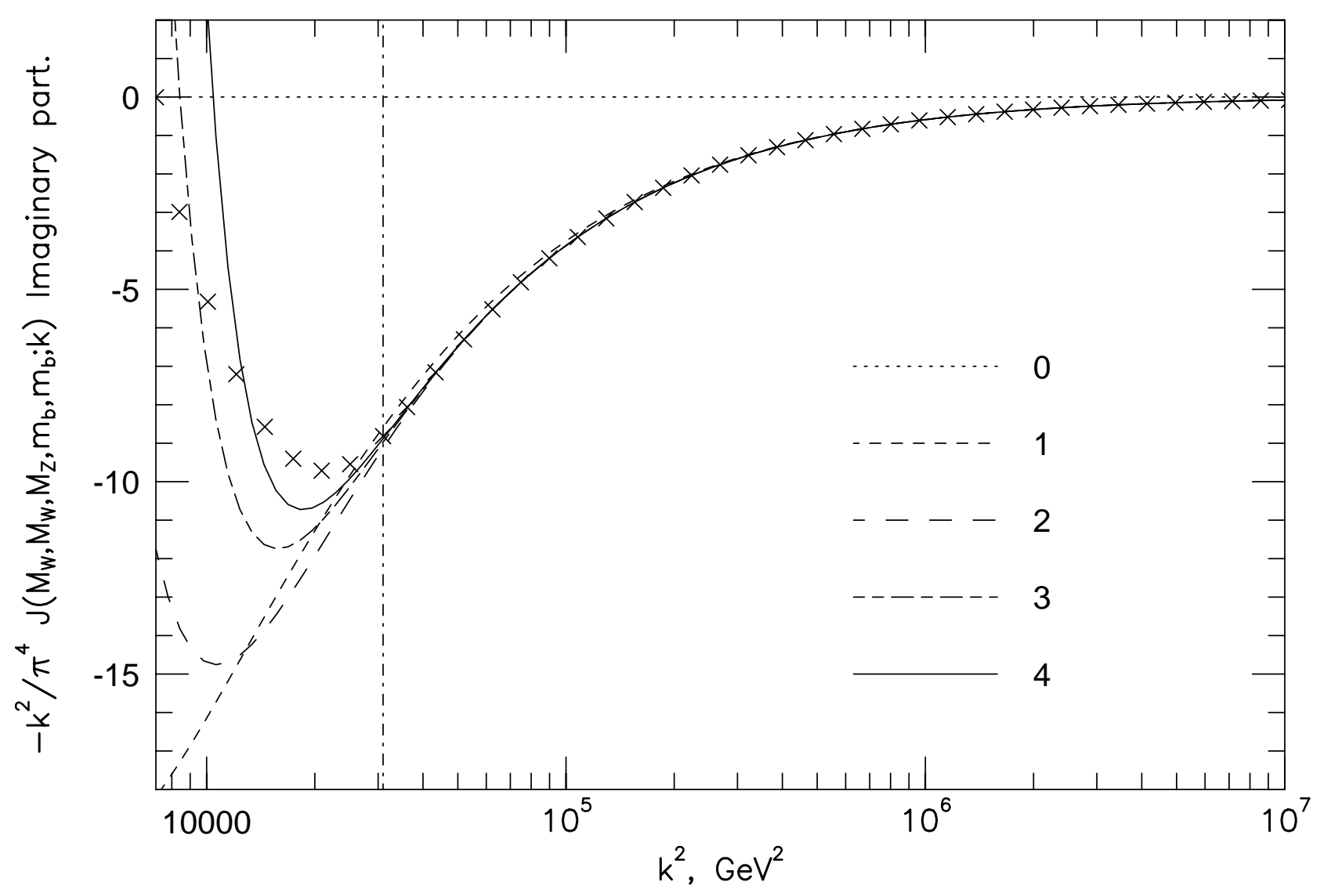


Fig. 4a

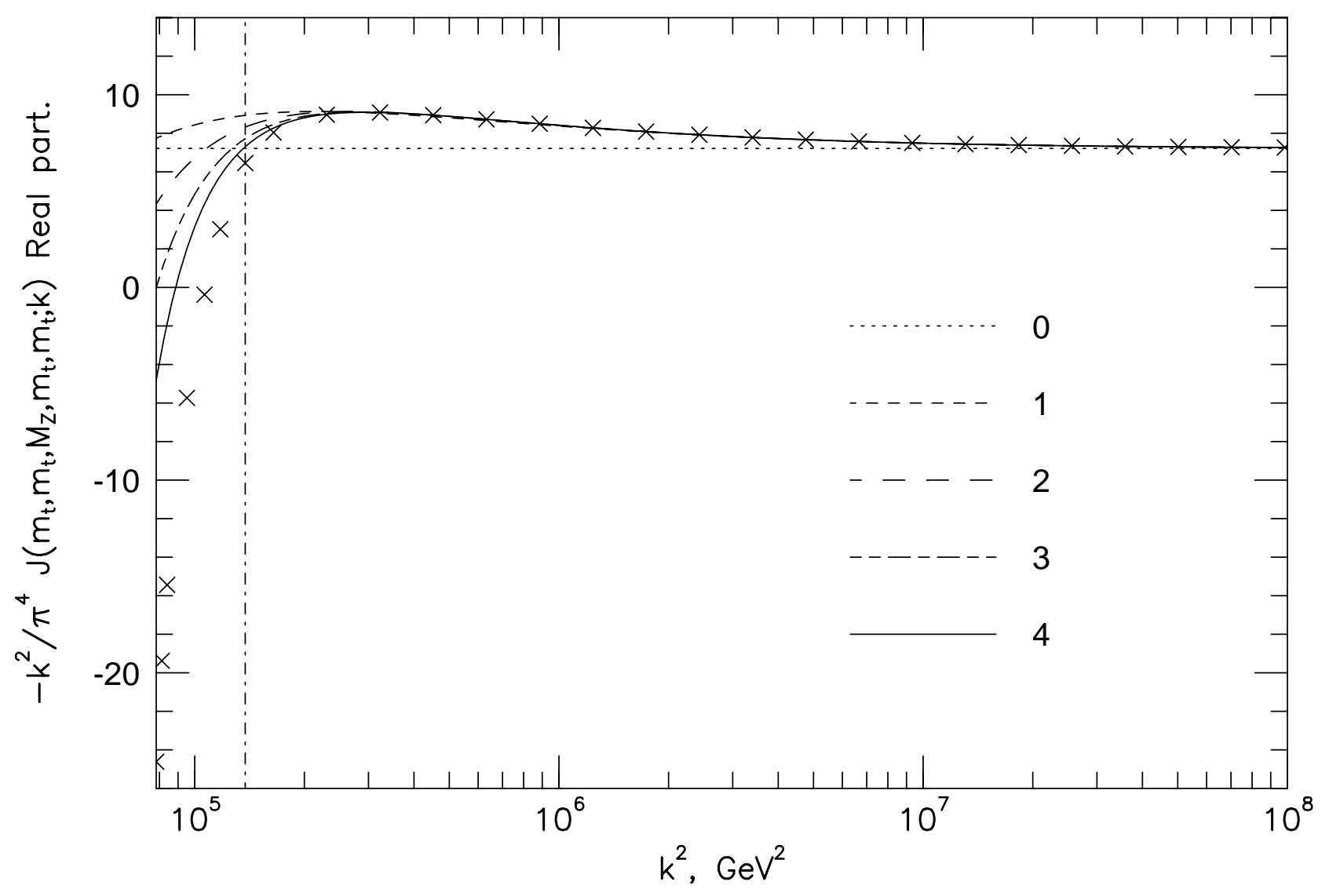

Fig. 4b

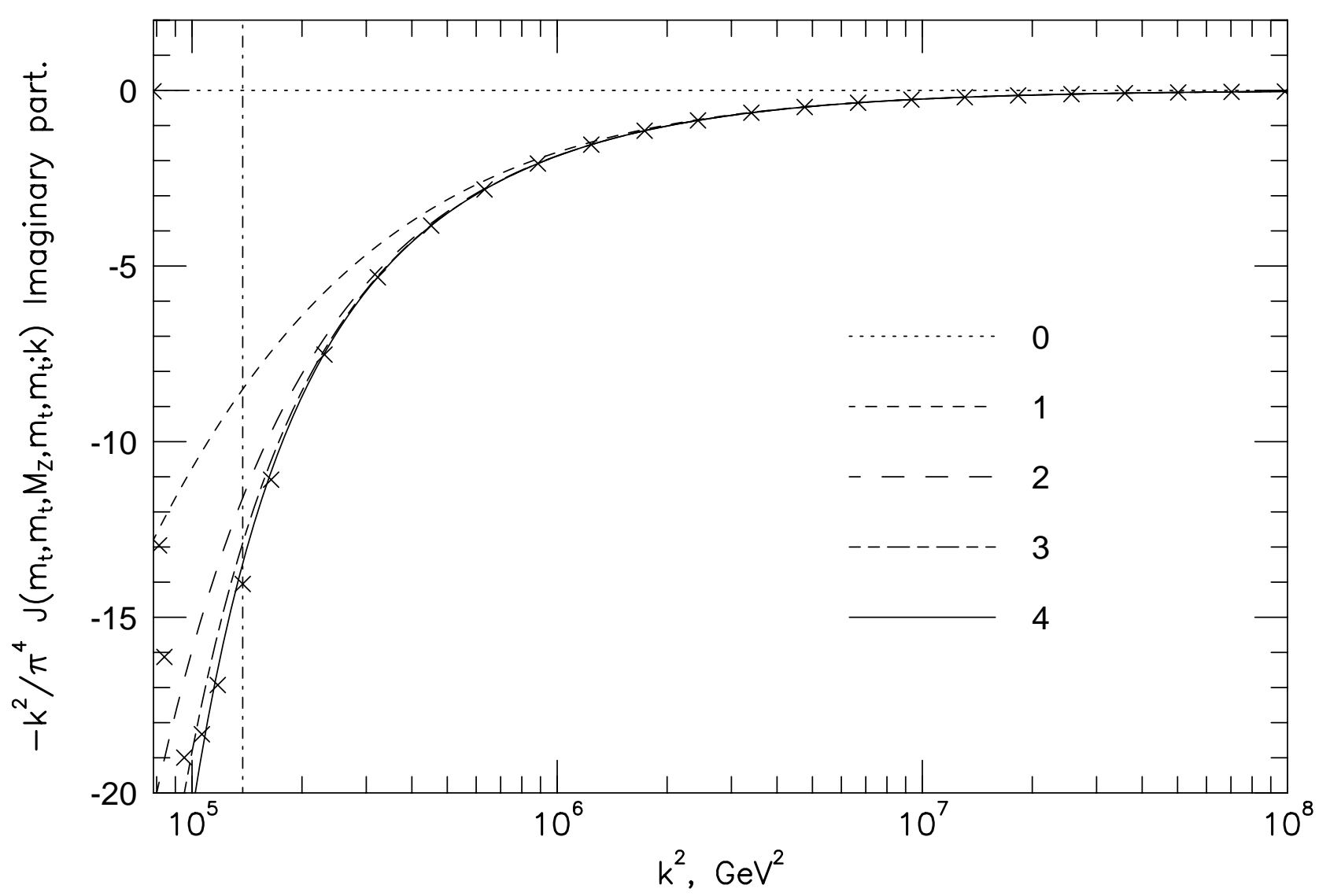


Fig. $5 a$

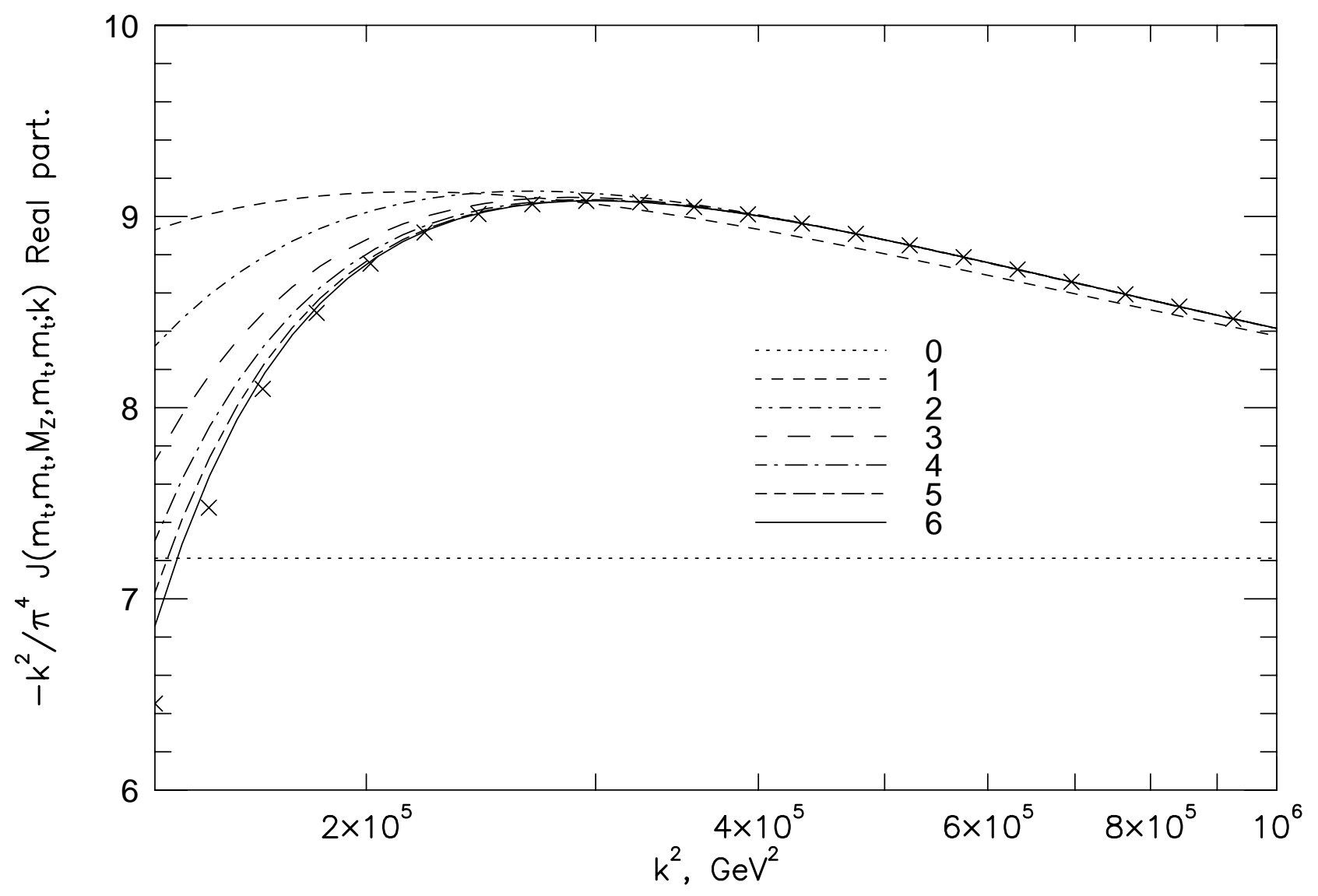

Fig. 5b

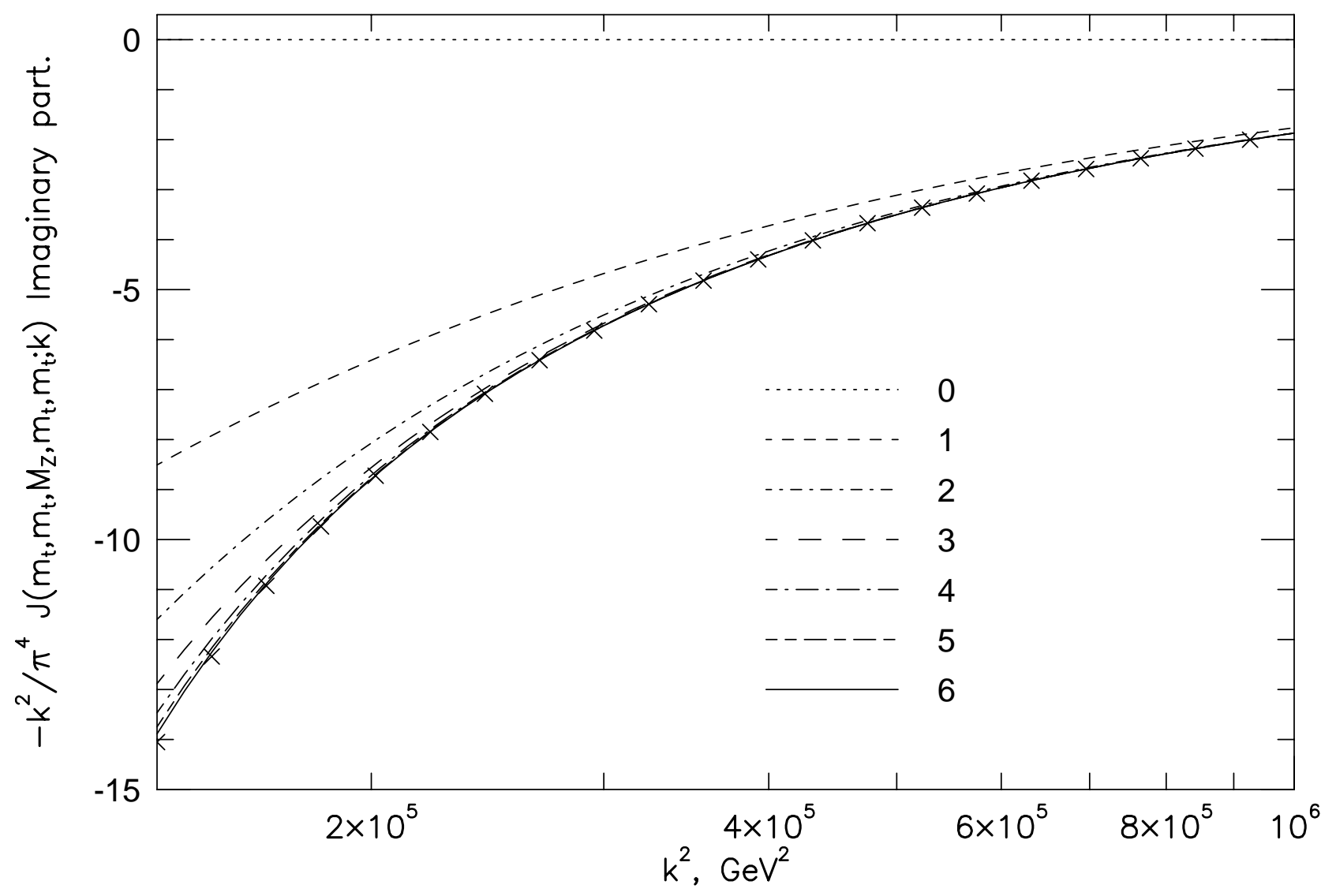

